\title{
Study Of On-Line Inspection Method Of Oversize And High Precision Bearing
}

\author{
Zhuangya ZHANG ${ }^{1}$, Mingli $\mathrm{LI}^{2}$,Xiangdong $\mathrm{Chen}^{2}$, Yumin Bao $^{2}$ \\ 1Henan University of Science and Technology, Luoyang, 471003, China \\ 2 Luoyang LYC bearing co., ltd Luoyang, 471003, China
}

Keywords: Oversize bearing; on-line inspection; data acquisition; error separation.

\begin{abstract}
According to high precision and oversize bearing's characteristics of large size, heavy weight and high precision, on-line inspection method based on contact measuring method is proposed. This inspection method uses contact double rotation probe and separates rotation error of machine tool through error separation technology, which realizes the continuous on-line measurement of inner and outer diameters, roundness and cylindricity of oversize bearing. Take the spindle bearing of large-scale CNC horizontal lathe for example, the inspection result shows that the precision meets the P4 standard, which satisfies precision test as well as provides theoretical foundation and technique support for the improvement of the accuracy of the oversize bearing.
\end{abstract}

\section{Introduction}

Equipment manufacturing industry is the fundamentality of the progress of national economy and the construction of national defense of a country ${ }^{[1,2]}$. As the basis of equipment manufacturing industry, the manufacturing level of machine tool may decide the technological level of the equipment manufacturing industry of a country ${ }^{[3,4]}$. The precision of the bearing of the machine tool spindle is a key contributor to the precision of the machine tool spindle, and the performance of the bearing directly affects the rotating speed, rotation accuracy, rigidity, anti-vibrating and cutting functions, noise, temperature rising and thermal deformation of the machine tool, then influences the accuracy of the mechanical parts processed and produced by machine tool. The current precision inspection methods in China for inner and outer diameters, roundness and cylindricity of the bearing are mechanical method, projection method, interferometry, computer vision technology $[5,6]$, laser technique ${ }^{[7,8]}$, etc.The premise of ensuring bearing's accuracy is to test it timely and effectively, however, there might not be an appropriate online testing method, while a majority of the manufacturers ensure accuracy by Machine tool to substitute actual inspection. For that reason, in order to guarantee the machining accuracy of high precision and oversize bearing, this article is based upon contact measuring method, using contact double rotation probe and separating rotation error of machine tool by error separation technology, which achieves the continuous on-line measurement of inner and outer diameters, roundness and cylindricity of oversize NC machine tool's spindle bearing.

\section{On-line inspection system for Oversize bearing}

According to the characteristic and technical index requirement of Oversize bearing, online monitoring system is composed of mechanical unit, data acquisition unit, control unit and human-machine interaction unit, system structure diagram is shown in Figure 1.

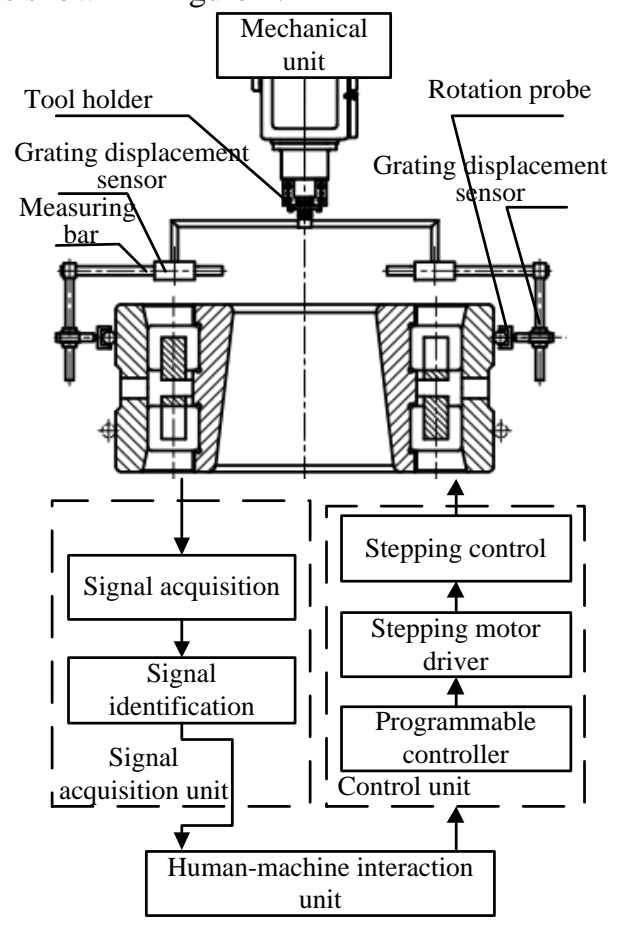

Figure 1: On-line inspection system

Mechanical unit mainly realizes the two kinds of motion during the measurement process, which include the rotation of the measured bearing and the movement up and down of probe in relation to measured bearing inner hole surface or outer circle surface, so as to realize the measurement of bearing inner hole and different section. Measuring mechanism mainly uses two high-accuracy rotary probe and relative measurement method, so as to realizes the mutual compensation of two readings. When measuring, the mechanical unit is installed on the machine tool holder, the measured bearing is installed on the machine tool working platform. The rotation of the measured bearing is realized by machine tool working platform and the 
movement up and down of probe in relation to measured bearing inner hole surface or outer circle surface is completed by machine tool holder.

Data acquisition unit mainly realizes acquisition and conditioning of the measurement signal, the displacement signal detection of measured bearing is collected by the displacement sensor, converting to corresponding electrical signal, and then by the differential amplify, shape, subdivision and direction discrimination, in order to meet the requirements of data acquisition.

Control unit is based on the detection requirement, PLC sends commands to the stepping motor driver, and the driver controls the speed and displacement of stepping motor by controlling the electric current, thus driving the movement up and down of the probe.

Human-machine interaction unit mainly realizes data management, system setting, parameter setting, real-time control and other auxiliary functions.

\section{Double rotation probe error separation}

In the process of measuring, as a result of the movement of the machine tool, it will inevitably bring the motion error of machine tool to measurement result. Therefore, we must use error separation technique to separate.

The first part of the method of double rotation probe error separation is shown in Figure 2 (a), double rotation probe acquire signal $V_{1}(\theta)$ and $\mathrm{V}_{2}(\theta)$ respectively.

$$
\left\{\begin{array}{l}
V_{1}(\theta)=s(\theta)+e(\theta) \\
V_{2}(\theta)=s(\theta-\pi)-e(\theta)
\end{array}\right.
$$

After fourier transformation, the type (1) is transformed into the fllowing formula (2):

$$
\left\{\begin{array}{l}
F_{1}(\theta)=F_{s}(n)+F_{e}(n) \\
F_{2}(\theta)=F_{s}(n) e^{-j n \pi}-F_{e}(n)
\end{array}\right.
$$

According to the superposition type $C_{1}=V_{1}(\theta)+V_{2}(\theta)$, the signal of $e(\theta)$ is eliminated. The corresponding Fourier transform as follows:

$$
\begin{gathered}
F_{e 1}(\theta)=F_{s}(n)\left(1+e^{-j n \pi}\right) \\
W_{1}(n)=\left(1+e^{-j n \pi}\right)=2 \cos (n \pi / 2)
\end{gathered}
$$

$W_{1}(n)$ is harmonic weight function. By the formula (3) and (4), The first part output of the method of Double rotation probe error separation only contains even harmonics, while the odd harmonics are inhibited.

The second part output of the method of Double rotation probe error separation is shown in Figure 2 (b). The measurement of first round to get signal $V_{1}(\theta)$, and the workpiece is rotated 180 degrees in the second round to get the singal $V_{2}(\theta)$, the form is as follows:

$$
\left\{\begin{array}{l}
V_{1}(\theta)=s(\theta)+e(\theta) \\
V_{2}(\theta)=s(\theta-\pi)+e(\theta)
\end{array}\right.
$$

The fourier transform of the type (5) is transformed into the fllowing formula (6).

$$
\left\{\begin{array}{l}
F_{1}(\theta)=F_{s}(n)+F_{e}(n) \\
F_{2}(\theta)=F_{s}(n) e^{-j n \pi}+F_{e}(n)
\end{array}\right.
$$

According to the superposition type $C_{1}=V_{1}(\theta)-V_{2}(\theta)$, the signal of $e(\theta)$ is eliminated. The corresponding Fourier transform as follows:

$$
\begin{gathered}
F_{e 2}(\theta)=F_{s}(n)\left(1-e^{-j n \pi}\right) \\
W_{2}(n)=\left(1-e^{-j n \pi}\right)=2 \sin ^{2}(n \pi / 2)
\end{gathered}
$$

So the second part output of the method of double rotation probe error separation only contains odd harmonics, while the even harmonics are inhibited. The composite of the two parts of the outputs has constituted the double rotation probe output

$$
F_{D}(n)=F_{e 1}(n)+F_{e 2}(n)
$$

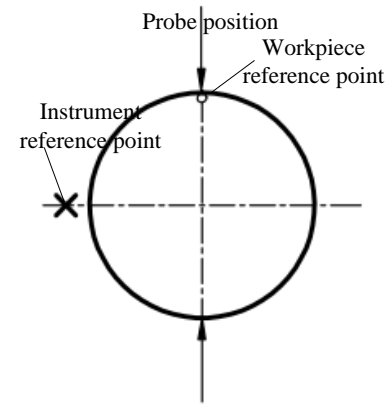

(a)
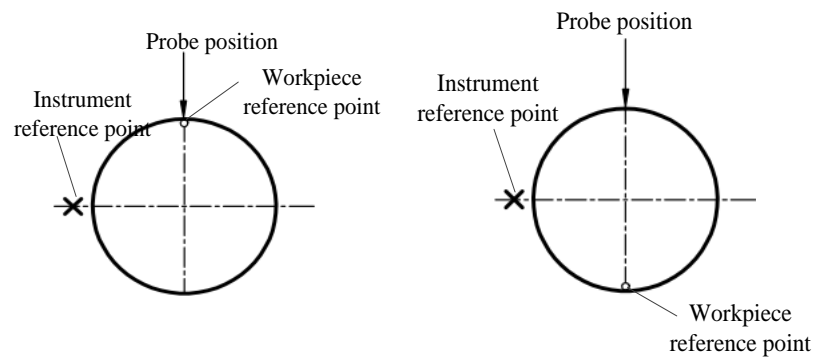

(b)

Figure 2: Double rotation probe error separation

\section{The bearing inner and outer diameter, roundness and cylindricity Measurement}

\subsection{The bearing inner and outer diameter Measurement}

Two point method was used to measure bearing inner and outer diameter, as shown in figure 3.

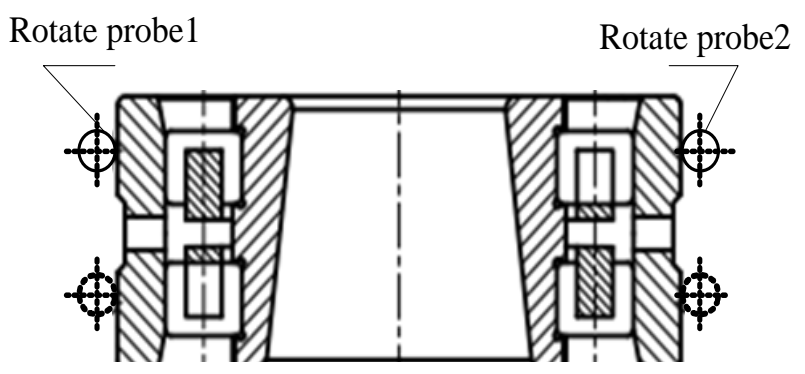

Figure 3: The bearing inner and outer diameter Measurement The bearing is measured at different radial measurement 
plane, at the same radial measurement plane, the machine tool worktable rotate more than a circle, though double rotation probe to measure the radial measurement plane's maximal outer diameter $D_{\text {spmax }}$, maximal inner diameter $d_{\text {spmax }}$ and minimum outer diameter $D_{\text {spmin }}$, minimum inner diameter $d_{\text {spmax }}$, then according to the definition of dimensional tolerance to calculate the single plane mean outer diameter deviation $\Delta_{D m p}$, mean inner diameter deviation $\Delta_{d m p}$, outer diameter variation $V_{D s p}$, inner diameter variation $V$ dsp. For several radial measurement plane of the same measure, several groups $D_{\text {spmax }}, d_{\text {spmax }}, D_{\text {spmin }}, d_{s p \min }, \Delta_{D m p}, \Delta_{d m p}, V_{D s p}$, $V_{d s p}$ can be obtained.

From several groups single plane's maximal outer diameter Dspmax and maximal inner diameter $d_{\text {spmax }}$, can find out the maximum value $D_{\text {spmax }}$ and $d_{\text {spmax }}$, then to calculate the maximal outer diameter deviation $\Delta_{D \text { smax }}$ and maximal inner diameter deviation $\Delta_{d s m a x .}$ From several groups single plane's minimum outer diameter $D_{\text {spmin }}$, minimum inner diameter $d_{\text {spmin }}$, can find out minimum value $D_{\text {spmin }}$ and $d_{\text {spmin }}$, which to calculate the maximal outer diameter deviation $\Delta_{D s m i n}$ and maximal inner diameter deviation $\Delta_{D \text { smin.. }}$ From several groups single plane's minimum outer diameter $D_{\text {spmin }}$, minimum inner diameter $d_{\text {spmin }}$, can find out minimum value $D_{\text {spmin }}$ and $d_{\text {spmin }}$ to calculate the maximal outer diameter deviation $\Delta_{D \text { smin }}$ and maximal inner diameter deviation $\Delta_{D \text { smin.. }}$ After that according to the definition of dimensional tolerance can calculate the bearing maximal mean outer diameter deviation $\Delta_{D m p}$, maximal inner mean diameter deviation $\Delta_{d m p}$, outer diameter variation $V_{D m p}$, inner diameter variation $V_{d m p}$. The Measurement of bearing inner and outer diameter relations as shown in figure 4.

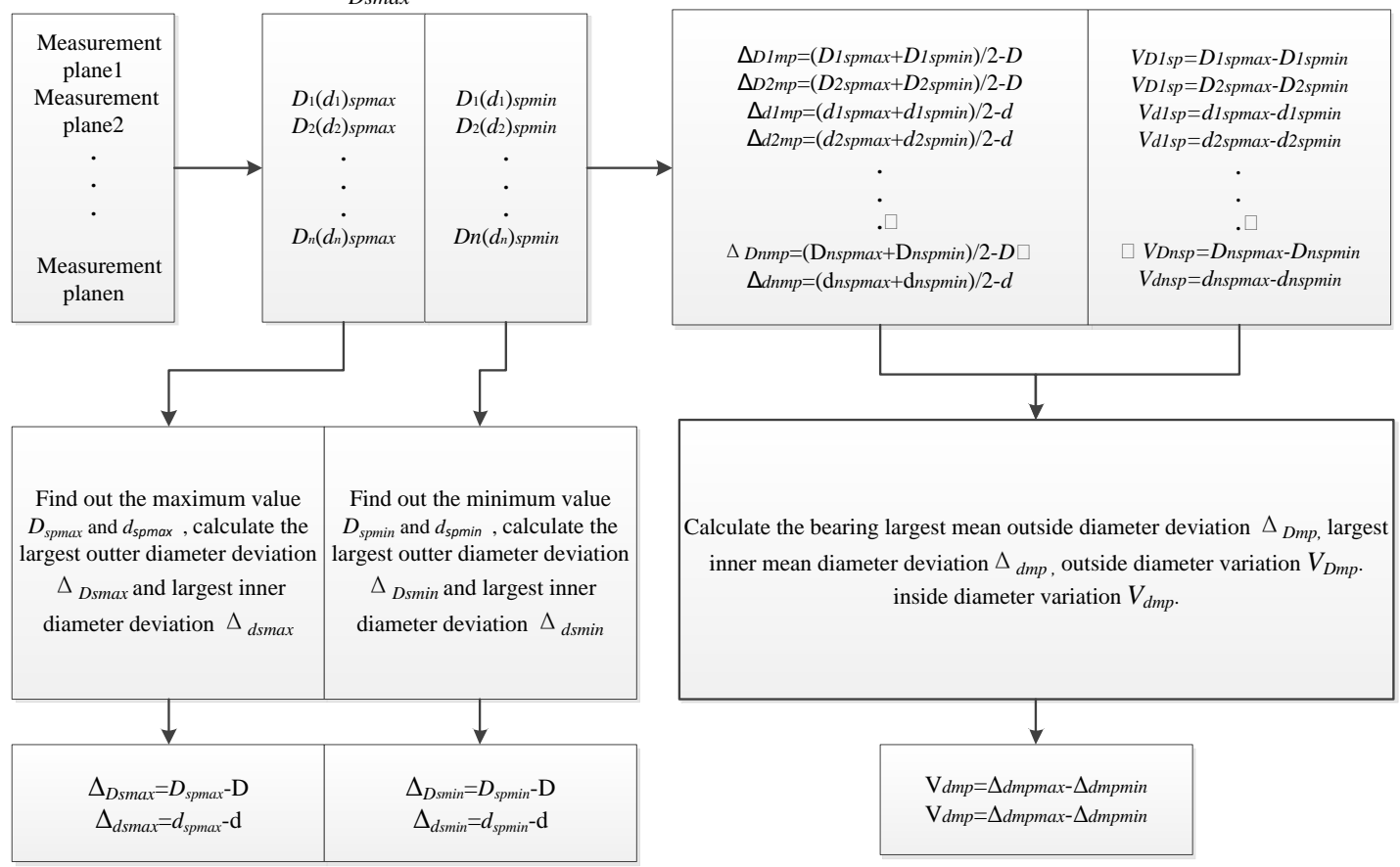

Figure 4: The measurement of bearing inner and outer diameter relations

\section{2 The bearing roundness and cylindricity Measurement}

Bearing roundness measurement variable radius measurement method, side head at one half of the width of the ring groove, in vertical direction on raceway plain wire measurement, The radial measurement approach was used to measure bearing roundness, the double rotation probe at one half of the width of the bearing ring and vertical raceway plain line, measurement principle is shown in figure 5.

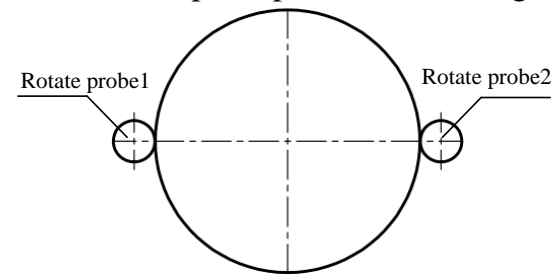

Figure 5: Bearing roundness measuring
Double rotation probe are installed in a straight line, and perpendicular to the axis of the bearing measuring , measuring direction and measure standard in a straight line, which help to eliminate the abbe error.

To reduce the measurement time, improve the accuracy of measurement, double rotation probe test sampling points at the same time, set the numbers of sampling points are $\mathrm{n}(\mathrm{n}$ is even, $i=1, \ldots, n)$, rotate probe 1 and 2 samples values is $\delta_{1 i}$ and $\delta_{2 i}$, the radius of the deviation is:

$$
\left\{\begin{array}{l}
\Delta_{i}=\frac{1}{2}\left(\delta_{1 i}+\delta_{2(i+n / 2)}\right), 1 \leq i \leq \frac{n}{2} \\
\Delta_{i}=\frac{1}{2}\left(\delta_{1 i}+\delta_{2(i-n / 2)}\right), \frac{n}{2} \leq i \leq n
\end{array}\right.
$$

According to the radius deviation, the bearing roundness error can be evaluate by minimun zone method, minimum two-multiply law and minimum circumscribeb circle. In the same way, by measuring the roundness error of the multiple 
bearing radial measurement plane, the bearing cylindricality error can be evaluate by minimum zone method (as shown in figure 6 ) and minimum circumscribeb circle.

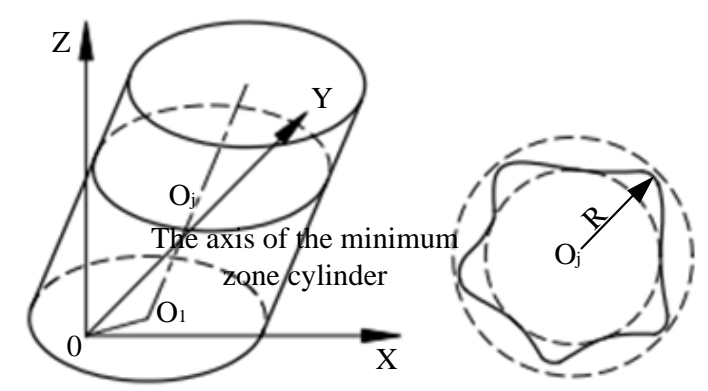

Figure 6: The minimum zone of cylindrical evaluation

\section{Experiment validation}

Taking Oversize and High Precision Double Row Roller bearing which used in 400 tons large-scale CNC horizontal lathe as example, as shown in figure 7, the test results are shown in table 1 , the inspection result shows that the precision meets the $\mathrm{P} 4$ standard, which satisfies precision requirement.

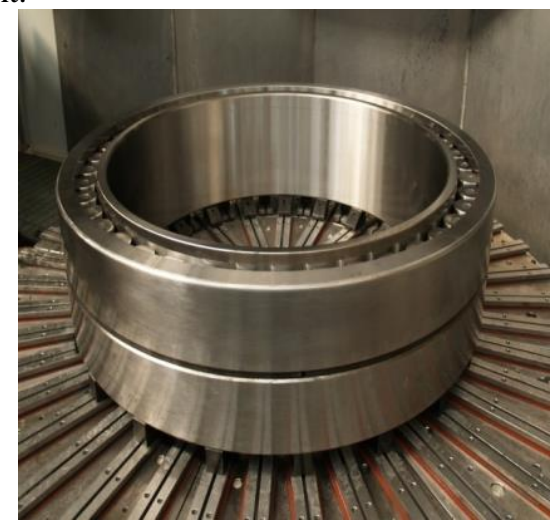

Figure 7: Large heavy load and high precision Double Row Roller bearing

Table1 Accuracy of measuring results

\begin{tabular}{|c|c|c|c|c|}
\hline & \multirow{2}{*}{$\begin{array}{c}\text { Measuring } \\
\text { item }\end{array}$} & $\begin{array}{c}\text { Standard } \\
\text { values }(\mu \mathrm{m})\end{array}$ & \multicolumn{2}{|c|}{$\begin{array}{c}\text { Measuring } \\
\text { value }(\mu \mathrm{m})\end{array}$} \\
\cline { 4 - 5 } & $\Delta_{d m p}$ & $+40 \sim 0$ & +18 & +22 \\
\hline 1 & $\mathrm{~V}_{d s p}$ & $\leq 11$ & 8 & 6 \\
\hline 2 & $\Delta_{d l m p} \sim \Delta_{d m p}$ & $+11 \sim 0$ & +5 & +6 \\
\hline 4 & $\Delta_{D m p}$ & $0 \sim-35$ & -20 & -13 \\
\hline 5 & $\mathrm{~V}_{D s p}$ & $\leq 15$ & 10 & 9 \\
\hline 6 & & & & \\
\hline
\end{tabular}

\section{Conclusion}

According to high precision and oversize bearing's characteristics of large size, heavy weight and high precision, on-line inspection method based on contact measuring method is proposed, this inspection method uses contact double rotation probe and separates rotation error of machine tool through error separation technology, which realizes the continuous on-line measurement of inner and outer diameters, roundness and cylindricity, which provides theoretical foundation and technique support for the improvement of the accuracy of the oversize bearing.

\section{Acknowledgements}

The project is supported by key research project of Henan province of China (Grant No. 152102210281), Educational Commission of Henan Province of China (Grant No. 16A460017) and Youth Foundation of Henan University of Science and Technology (Grant No.2015QN005 ).

\section{References}

[1] Zhang Danyang, Chen Yang. Study on Development Level and Mode of Equipment Manufacturing Industry in China. The Journal of Quantitative \& Technical Economics, 07,99-114,(2014).

[2] Sun Tao, Zhao Shukuan, Qiao Zhuang. Research on Current Situation of Transform and Promotion and Development Countermeasures of Equipment Manufacturing Industry in our Country. Journal of Industrial Technological Economics, 05,38-41, (2011).

[3] YANG Shuzi, Ding Han, Li Bin. State Key Lab of Digital Manufacturing and Equipment and Technology. Machine Building \& Automation,01,1-5, (2011).

[4] Wang Taiyong, Qiao Zhifeng, Han Zhiguo, et al. Development Trends of High-end NC Equipement [J]. China Mechanical Engineering,10,1247-1252+1259, (2011).

[5] YANG Min, YE Bang-yan, MU Li, YAN Jun. CCD is applied to On-line Inspection of Round Spindle' $\mathrm{s}$ Roundness. Mechanical Engineer, 04,38-40, (2003).

[6] CHEN Xiang-wei, WANG Long-shan, LIU Qing-min, CUI Zhi. Research on Measurement of Roundness Errors Based on CCD Image. Semiconductor Optoelectronics, 04,313-316, (2004).

[7] Shi Jiliang, Dong Lijun, Liang Guoxing. Acquisition method of roundness error data based on MATLAB image processing $[\mathrm{J}]$. Journal of Chinese Agricultural Mechanization,2011,02,257-261,(2011).

[8] MA Zi-ping, KANG Bao-sheng. Image retrieval for color images based on circularity measure of complex moment. Application Research of Computers,07,27432746, (2012). 